\title{
Measurement and Numerical Simulation of Ground and Subgrade Vibrations of Beijing Urban Rail Line 13
}

\author{
Futong Wang $\mathbb{D}^{1},{ }^{1}$ Leilei Yan, ${ }^{1}$ Qi Jiang, ${ }^{1}$ and Xiaxin Tao $^{2,3}$ \\ ${ }^{1}$ School of Civil Engineering, Heilongjiang University, Xuefusandao 36, Harbin, China \\ ${ }^{2}$ School of Civil Engineering, Harbin Institute of Technology, Haihe 202, Harbin, China \\ ${ }^{3}$ Institute of Engineering Mechanics, China Earthequake Administration, Xuefu 29, Harbin, China \\ Correspondence should be addressed to Futong Wang; 2002208@hlju.edu.cn
}

Received 1 September 2019; Revised 5 January 2020; Accepted 1 February 2020; Published 24 February 2020

Academic Editor: Chiara Bedon

Copyright ( $\odot 2020$ Futong Wang et al. This is an open access article distributed under the Creative Commons Attribution License, which permits unrestricted use, distribution, and reproduction in any medium, provided the original work is properly cited.

Urban rail transit is an effective way to deal with the problem of traffic congestion in major cities. Trains travel through dense residential and commercial areas, providing convenient transportation while also result in vibration problems in the surrounding environment. Long-lasting vibrations result in disturbance to people's sleep, malfunction of sensitive equipment, and even damage to heritage buildings. Compared with elevated and tunnel sections, ground surface urban railway generates vibrations and propagates to the surroundings via a more direct path in the form of surface waves, which makes the environmental problem more prominent. Due to the complexity of the train-track-ground system, the characteristics of the vibration propagation and attenuation are yet to be revealed. In this paper, we investigate the vibration of the ground and the subgrade next to the Beijing Urban Rail Line 13 by a field measurement combined with a mathematical model. The duration of ground vibration is divided into two parts: the train passing time and the Doppler effect-related tailing part. Through a regression analysis of the duration, the train passing time is identified and the train traveling speed is estimated. The attenuation relationship of ground vibration intensity is expressed by a piecewise function. In the subgrade, the vibration intensity of particle decays with increasing depth and the stress decay rate is faster than that of the acceleration. The dynamic wheel/rail interaction behaves stationary and periodic, and the magnitude fluctuates up and down with the quasi-static axle weight. The intensity attenuation relationship fitted in this paper provides a basis for designing new lines and renewing old lines and can be used as a reference for the development of vibrationreduction technology. The simulated time history of the wheel-rail force provides an excitation sample for further model experiments and numerical simulation. The proposed train speed identification method may be useful for parameter identification of moving sources such as ships, automobiles, and airplanes.

\section{Introduction}

The urban rail transit has the characteristics of large passenger capacity, fast speed, and excellent punctuality. It, therefore, has become one of the most effective means to cope with the traffic dilemma in big cities. Trains travel through dense residential and commercial areas, providing a kind of convenient transportation, causing environmental vibration problems. During operation, the train-induced vibration propagates in the form of waves through the track, the subgrade, and the foundation soil, causing a long-term vibration in the ground and the buildings nearby. This environmental problem has become increasingly prominent, and the need for vibration reduction is quite urgent. The development of effective vibration-reduction techniques relies on a good understanding of the behaviors of the vibration propagation.

It is undoubtedly necessary to conduct in situ measurements, theoretical analyses, and numerical simulations to investigate the dominant frequency bands and spatial distribution characteristics of the ground vibration. The results may provide an essential basis for the prediction of the vibration when planning new lines and also provide a necessary reference for developing the vibration isolation when updating existing lines.

Studies on the vibration of rock and soil next to railway lines are mostly devoted to high-speed, heavy-haul, or 
Intercity railways. Among them, Xia et al. [1] measured the Shenyang-Shanhaiguan Railway in China and found that the ground vibration level may approach as high as $107.5 \mathrm{~dB}$ near a pier foot. Excessive vibration was also recorded by an in situ test [2] near the Beijing-Guangzhou railway, where the vertical vibration levels have exceeded the allowance of Chinese code for the environmental vibration. Gao et al. [3] examined the acceleration characteristics of the surrounding areas of the Qin-Shen Railway in China and discussed the effect of driving speed on the vibration. Based on the numerical results of an elevated railway, Mao et al. [4] pointed out that the vibration significantly increases either the vehicle speed increases or the shear wave velocity of soil decreases. The response of a sandy subgrade under the groundwater table was reported by Chang et al. [5], who found that the low-frequency response is of the dominant range of the vertical vibration. Auersch [6] investigated the Berlin-Hanover high-speed rail and indicated that the ground vibration is mainly caused by dynamic loads due to irregularities of the wheels and the track. In contrast, the gravity-induced quasi-static load is not essential except for the very-low-frequency band of the vibration in the vicinity of the track. Feng et al. [7] established a three-dimensional time-domain finite element model for a high-speed railway subgrade. They discussed the distribution of the displacement amplitude under the high-speed moving load, indicating that high-frequency contents have a stronger influence on the subgrade and less impact on the far-field vibration. The seasonal frozen effect on the ground vibration of the Harbin-Daqing Railway in China was studied by Tian et al. [8]. They pointed out that the seasonally frozen soil layer has a magnifying effect on the vertical vibration of the subgrade. Similar results were reported by Dong and Wu [9] according to an experiment for the China Da-Qin heavyhaul railway, where they indicated that this effect occurs at far-field as well. Recently, a field measurement, as well as a finite/infinite element simulation for the Shanghai-Hangzhou high-speed rail, was reported by Tang et al. [10]. They identified the characteristic frequency of the subgrade vibration and summarized the spectrum distribution characteristics, the attenuation behavior, and the train speed effect. Hu et al. [11] measured the China Shuo-Huang heavy-haul railway and presented a numerical model to study the attenuation of the vibration velocity.

The research on the ground vibration of the urban rail transit is mostly concentrated in underground tunnel segments [12, 13]. Obviously, because of the different propagation paths of vibration, the urban railway on the ground surface is quite different from the underground or viaduct sections. Compared with those of the intercity railway, the trains of the surface urban railway has smaller axle loads with a lower travel speed but is closer to the living and working areas of cities. At present, there are relatively few studies on the vibration caused by the ground surface urban train. Cui et al. [14] and Wang et al. [15] reported an experimental investigation to the surface traveling section of the Beijing Urban Rail Line 13. Based on the observed data, they identified a dominant frequency range and proposed an attenuation relationship for the ground-borne vibration.
However, the number of observation points are limited and are not able to cover the entire wavefield intensively. For surface urban rail transit, the ground vibration, as well as that propagating deep in the foundation soil, still needs to be studied extensively.

The purpose of this paper is to extend the works in $[14,15]$, further exploring the vibration and propagation characteristics of the subgrade and ground of the Beijing Urban Rail Line 13. We focus on the combination of the field measurement and numerical simulation to reveal the features of the vibration duration and intensity. First, we briefly introduce the test location, the type of the train, the observation configure, and the profile of the soil. Then, we outline the mathematical modeling approach and verify the performance of the model by the experimental records. Later, we identify the train speeds from the durations of acceleration records, and based on the results of the experiment and the simulation, we evaluate and revise the attenuation equation of Cui et al. [14]. Finally, the variation of vibration amplitude with depth is revealed deep in the foundation soil. Furthermore, a stochastic sample of the wheel/rail interactive force is put forward for future studies.

\section{Methodology}

2.1. Outline of the In Situ Test. The test field was selected in a close-track area of Line 13 of Beijing Urban Rail. According to the GPS data, the latitude and longitude of the site are $40.4118^{\circ} \mathrm{N}$ and $116.2096^{\circ} \mathrm{E}$, which indicates that the test location is between the Huilongguan Station and the Huoying Station. The train is produced by CRRC Changchun Railway Co., Ltd., and is composed of 4 DKZ5 vehicles. The basic dimensions are shown in Figure 1. Each vehicle is supported by two bogies, each with two axles. The test section has a classic ballast embankment. The statistical characteristics of the wheel/rail random irregularity were obtained by an inverse analysis [16]. One vertical irregularity sample is shown in Figure 2, where $x$ represents the coordinate along the track direction.

In order to reveal the geotechnical conditions of the test site, a geotechnical engineering survey was carried out. Figure 3 illustrates the profile of the soil. According to borehole samples, the surface layer is a thin layer of artificial fill, beneath which is a thick silt layer with the bottom buried $15 \mathrm{~m}$ deep. Next to the silt are a fine sand layer and a deep soil of silty clay. Besides, the shear wave velocity structure of the site was measured by the suspended wave velocity logging method, and the obtained results are shown in Figure 3 as well.

Five observation points were arranged on the free ground beside the track, aligned with a line perpendicular to the track. Their position in the $y$-direction is also shown in Figure 3. The distances from the midline of the track to the observation points are $10 \mathrm{~m}, 20 \mathrm{~m}, 40 \mathrm{~m}, 50 \mathrm{~m}$, and $60 \mathrm{~m}$, respectively. A three-component accelerometer was placed at each point to form an observing array. When a train was passing, the array recorded the acceleration time histories at a sampling frequency of $200 \mathrm{~Hz}$. Altogether, sixteen passes were recorded during the test. 


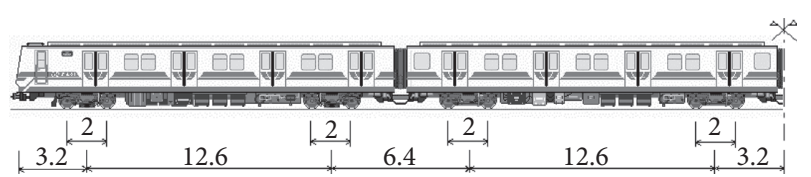

FIGURE 1: Schematic diagram of the urban train in the test (unit: $\mathrm{m}$ ).

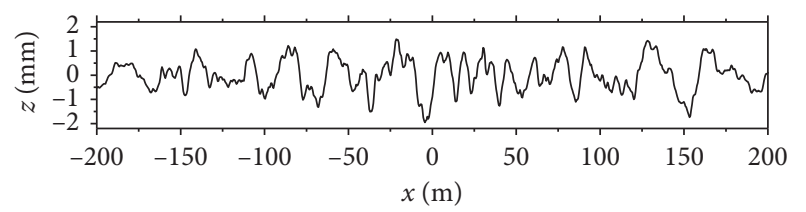

FIgURE 2: A stochastic sample of wheel/rail irregularities of the Beijing Urban Rail Line 13.

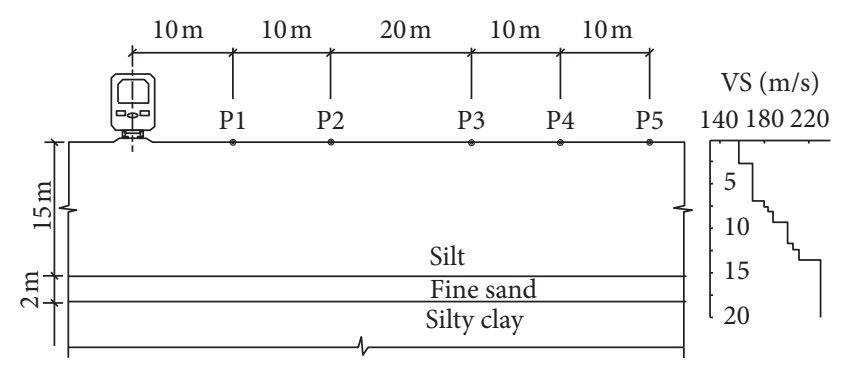

Figure 3: Cross section of the field test.

Observed signals inevitably contain background noise, especially at measurement points that are far from the track. To increase the signal-to-noise ratio, we use the auto-cross PSD method [17] to remove the background vibration. The basis of this method is that the signal and the background vibration have the cross-correlation because they propagate in the same site. It has been found that the auto-cross PSD method is more suitable for the traffic-induced environmental vibration problem than other methods such as filtering, PSD correction [18], and Fourier spectrum correction [19].

\subsection{Semianalytical Model of Coupled Train-Track-Ground} Vibration. A train-induced wavefield is generally quite large, whereas any in situ test has a limit number of observation points, such that the intensiveness and the coverage of the measurement are not sufficient to reflect the whole picture of the wavefield. The in situ test above set up five observation points with spacing of 10 meters or 20 meters. All the vibration sensors were installed on the ground surface so that the responses inside the soil cannot be reflected simply by the experimental data. The advantage of numerical simulation is that it can produce some necessary supplement to an observation, providing values that are not directly available by the actual measurement. For the problem of the coupled train-track-ground system, the first author of the present paper established a semianalytical model based on the work of Sheng et al. [20]. Because of the extensive verifications [21-28], the model has been perfect for the simulation of train-induce vibration. The basic idea and fundamental formulation are outlined below. More details should be found in [21].

2.2.1. Mathematical Modeling. The train's model is a massspring-damper system consisting of multiple rigid blocks and spring dampers. A total of $M$ axles is considered in the whole train, whose transfer function matrix $\mathbf{H}_{w}$ [20] is obtained by applying a vertical harmonic excitation at each axle. The rail is represented by a Euler-Bernoulli beam with the bending stiffness of EI. Rail pads are simplified as a continuous distribution spring with the stiffness of $k_{p}$. Sleepers and ballast are simplified to a flat beam with zero-bending stiffness, with the mass per unit length $m_{s}$. The consistent mass approximation is applied to the embankment, with the mass per unit length of $m_{B}$ and the vertical stiffness of $k_{B}$.

According to the Fourier series theory, the wheel/rail irregularity in Figure 2 can be decomposed into a series of harmonic unevenness. For the $i$-th uneven component with the amplitude of $z_{i}$, the wavenumber of $2 \pi \beta$, and the wavelength of $1 /(2 \pi \beta)=\lambda_{i}$, the up and down vibration frequencies of the train is $\Omega_{i}=2 \pi c / \lambda_{i}$ when it moves at the speed of $c$. The up and down movements of the wheels result in a series of moving time-harmonic forces at wheel/rail contacts. Considering the compatibility at the wheel/rail contacts, we obtain a vector collecting all the amplitudes of the wheel/rail forces as follows:

$$
\mathbf{P}=-\left[\mathbf{H}_{R}+\mathbf{H}_{w}+\mathbf{K}_{h}^{-1}\right]^{-1} \mathbf{Z},
$$

where the element $P_{j}$ of the vector $\mathbf{P}$ is the $j$-th wheel/rail force amplitude; the vector $\mathbf{Z}=\left\{e^{i \Omega_{i} a_{1} / c}, e^{i \Omega_{i} a_{2} / c}, \ldots\right.$, $\left.e^{i \Omega_{i} a_{M} / c}\right\}^{\mathrm{T}}, i=\sqrt{-1}$, and $a_{1}$ to $a_{M}$ are the initial coordinates of the $M$ axles; the diagonal matrix $\mathbf{K}_{h}=\operatorname{diag}\left(k_{h 1}, k_{h 2}, \ldots, k_{h M}\right)$, $k_{h j}$ denoting the linearized Hertz stiffness of the $j$-th wheel/ rail contact; and $\mathbf{H}_{R}$ stands for the transfer function matrix of the track-ground subsystem. The element at the $l$-th row and $m$-th column of $\mathbf{H}_{R}$ is

$$
H_{l m}^{R}=w\left(a_{l}-a_{m}\right), \quad l, m=1, M,
$$

where $w\left(x^{*}\right)$ denotes a function defined on the abscissa $x^{*}=$ $x-c t$ moving with the train. The spatial Fourier transform of $w\left(x^{*}\right)$ can be obtained by

$$
\widetilde{w}(\beta)=\frac{\Delta_{1} \Delta_{2}-\Delta_{4}^{2} H}{\Delta_{1} \Delta_{2} \Delta_{3}-\Delta_{3} \Delta_{4}^{2} H-\Delta_{1} k_{p}^{2}},
$$

where $\Delta_{1}=1+k_{B} H-\omega^{2} m_{B} H / 3, \Delta_{2}=k_{p}+k_{B}-\omega^{2}\left(m_{s}+m_{B} / 3\right)$, $\Delta 3=E I \beta^{4}-\omega^{2} m_{R}+k_{p}$, and $\Delta_{4}=k_{B}+\omega^{2} m_{B} / 6 ; \omega$ are the angular frequencies of a particle in the track-ground subsystem, and

$$
\omega=\Omega i-\beta c .
$$

The function $H$ in equation (3) is the receptance of the subgrade and is calculated by

$$
H=-\frac{1}{2 \pi} \int_{-\infty}^{+\infty} \delta(\beta, \gamma) \frac{\sin \gamma b}{\gamma b} \mathrm{~d} \gamma,
$$

where $\delta(\beta, \gamma)$ denotes the transferred Green's function of multilayered half-space [22], $\gamma$ is the angular wavenumber in 
the $y$-direction (i.e., the horizontal direction perpendicular to the track), and $b$ is the half-width of the embankment bottom.

The wheel/rail force sequence $\mathbf{P}=\left(P_{1}, P_{2}, \ldots, P_{M}\right)$ results in the vibration of the train-track-ground system. From the perspective of an observer staying in the moving reference frame $\left(x^{*}=x-c t, y, z\right)$, all responses are harmonically changing over time with the same frequency $\Omega_{i}$. The vibration pattern in the wavenumber domain is written as

$$
\widetilde{U}_{P}(\beta, \gamma)=\widetilde{U}(\beta, \gamma) \sum_{l=1}^{M} P_{l} e^{-i \beta a_{l}},
$$

where $\widetilde{U}(\beta, \gamma)$ stands for the displacement pattern excited by a single time-harmonic load moving on the track [22]. For a particle in the fixed reference frame $(x, y)$, the Fourier spectrum of displacement is

$$
S\left(\omega ; \Omega_{i}\right)=\frac{-e^{i \beta x} U_{p}(\beta, y)}{c},
$$

where the function $U_{p}(\beta, y)$ is the inverse Fourier transform of $\widetilde{U}(\beta, \gamma)$ with respect to $\gamma$.

Under the condition of harmonic unevenness, the spectrum response can be obtained in terms of (7). By taking the unevenness amplitudes as weighting factors and superposing all the responses, we have the total spectrum response as

$$
S(\omega)=S_{0}(\omega)+\frac{\Delta \beta}{2 \pi} \sum_{i=-\infty}^{+\infty} S\left(\omega ; \Omega_{i}\right) z_{i},
$$

where $\Delta \beta$ is the sampling interval of $\beta$ and $S_{0}(\omega)$ denotes response to the quasi-static axle loads and should be calculated according to (7) by letting $\Omega_{i}=0$ and $P_{j}$ equal to the gravity allocated to the $j$-th axle.

Corresponding to the spectrum of the displacement (8), the velocity and acceleration spectra are $(i \omega) S$ and $-\omega^{2} S$, respectively. By the inverse Fourier transform of the spectra with respect to $\omega$, we obtain the stochastic responses in the time domain.

2.2.2. Model Reliability Verification. To verify the mathematical model above, we simulate the ground vibration at the five observation points of the in situ test. The required values of parameters are listed in Tables 1 and 2, where the train and the track parameters are based on the design documents and the soil parameters are from the geotechnical engineering survey.

Figure 4 shows the simulation results for the train of 7 : $40 \mathrm{am}$, with a driving speed of $22.72 \mathrm{~m} / \mathrm{s}$. The acceleration time histories of the model output are well consistent with the observed data, both in terms of amplitude and duration. These results show the reliable performance of the mathematical model of the coupled train-track-ground vibration in the time domain.

To further compare the frequency domain results, the time histories are converted to the vibration acceleration level (VAL) according to the Chinese national standard Urban Regional Environmental Vibration Measurement
Method [29]. The results are shown in Figure 5. It can be seen that the calculated results appear to be slightly overestimated at the low-frequency of $1.6 \mathrm{~Hz}$. For the vibration level above $1.6 \mathrm{~Hz}$, though, the numerical results are close to the experimental results at all the measurement points.

\section{Envelope Feature of Time Histories at Near- Field of Track}

Figure 6 shows a set of vertical acceleration time histories measured in the field, where five distinct groups can be seen in the curve of the nearest point P1. This was caused by the passing of the train's five groups of wheels. As shown in Figure 1, each vehicle has two bogies located at the front and rear ends. At the junction of two cars, the bogie at the rear end of the front vehicle is close to the bogie at the front end of the rear vehicle, which forms a group of wheels under the junction. The four-segment train has three junctions with three wheel-groups. Together with the front bogie of the first car and the rear bogie of the last car, there are a total of five wheel-groups. When the five groups successively pass through the observation section, the excited ground vibrations are recorded by the accelerometer at P1. Therefore, the group effect is clearly shown in the envelope of the first time-history in Figure 6. This effect is easily identified by the near-field $\mathrm{P} 1$ point, but for the far-field points at the distance of $20 \mathrm{~m}$ or further, vibrations from the wheel-groups interfere with each other, such that the group effect can hardly be seen in the time histories.

\section{Duration Feature of Ground Vibration}

Figure 6 also shows that, as the distance from the track increases, there is a significant increase in the vibration duration. For a time history $a(t)$ of an earthquake ground motion, Trifunac and Brady [30] proposed a method to calculate the duration:

$$
T_{d}=T_{2}-T_{1}
$$

where $T_{1}$ and $T_{2}$ are the moments corresponding to the ground motion energy $I(t)$ reaching $5 \%$ and $95 \%$, respectively, and the energy

$$
I(t)=\frac{\int_{0}^{t} a^{2}(t) \mathrm{d} t}{\int_{0}^{T} a^{2}(t) \mathrm{d} t}
$$

where $T$ is the end of the record. We use this method to calculate the duration of each curve of Figure 6 , and the results are shown in Figure 7. In the test, the other 9 trains heading towards Huoying station were also recorded. All the obtained three-component time histories are calculated according to equation (9), and the durations $T_{d}$ are listed in Table 3. Besides, the peak of each record (i.e., PGA) is also shown in the table.

Both Figure 7 and Table 3 show that, as the distance increases, the vibration duration appears to increase linearly. Therefore, we perform a linear regression on the experimental data in Figure 7 using the following equation: 
TABle 1: Model parameters of train and track for Beijing Urban Rail Line 13.

\begin{tabular}{lccc}
\hline \multicolumn{1}{c}{ Vehicle } & & \multicolumn{2}{c}{ Track structure } \\
Parameter & Value & Parameter & Value \\
\hline Car body mass & $5.0 \times 10^{4} \mathrm{~kg}$ & Mass per unit length of rails $m_{R}$ & $121 \mathrm{~kg} / \mathrm{m}$ \\
Car body pitch inertial & $1.5 \times 10^{6} \mathrm{~kg} \mathrm{~m}$ & Flexural rigidity of rails $E I$ & $1.3 \times 10^{4} \mathrm{kN} \cdot \mathrm{m}^{2}$ \\
Bogie mass & $3.0 \times 10^{3} \mathrm{~kg}$ & Hysteric damping ratio of rail $\eta_{R}$ & 0.005 \\
Bogie pitch inertial & $2.1 \times 10^{3} \mathrm{~kg} \mathrm{~m}$ & Stiffness per unit length of rail pad layer $k_{p}$ & $3.5 \times 10^{5} \mathrm{kN} / \mathrm{m}^{2}$ \\
Wheelset mass & $9.0 \times 10^{2} \mathrm{~kg}$ & Hysteric damping ratio of rail pad layer $\eta_{p}$ & 0.075 \\
Parallel stiffness of primary suspension & $2500 \mathrm{kN} / \mathrm{m}$ & Mass per unit length of sleeper layer $m_{s}$ & $416 \mathrm{~kg} / \mathrm{m}$ \\
Series stiffness of primary suspension & $1.0 \times 10^{12} \mathrm{kN} / \mathrm{m}$ & Mass per unit length of embankment $m_{B}$ & $2400 \mathrm{~kg} / \mathrm{m}$ \\
Parallel stiffness of secondary suspension & $1000 \mathrm{kN} / \mathrm{m}$ & Stiffness of unit length embankment $k_{B}$ & $1.45 \times 10^{4} \mathrm{kN} / \mathrm{m}^{2}$ \\
Series stiffness of secondary suspension & $1.0 \times 10^{12} \mathrm{kN} / \mathrm{m}$ & Damping ratio of embankment material $\eta_{B}$ & 0.05 \\
Viscous damping of primary suspension & $8.0 \mathrm{kN} \mathrm{s} / \mathrm{m}$ & Width of embankment bottom $2 b$ & $4.50 \mathrm{~m}$ \\
Viscous damping of secondary suspension & $6.0 \mathrm{kN} \mathrm{s} / \mathrm{m}$ & Hertz stiffness for two wheels $k_{h}$ & $2.4 \times 10^{6} \mathrm{kN} / \mathrm{m}$ \\
\hline
\end{tabular}

TABLE 2: Soil parameters.

\begin{tabular}{|c|c|c|c|c|}
\hline \multirow{2}{*}{ Layer thickness (m) } & \multicolumn{2}{|c|}{ Lamé constant $(\mathrm{MPa})$} & \multirow{2}{*}{ Mass density $\left(\mathrm{kg} / \mathrm{m}^{3}\right)$} & \multirow{2}{*}{ Shear wave velocity $(\mathrm{m} / \mathrm{s})$} \\
\hline & $\mu$ & $\lambda$ & & \\
\hline 4.0 & 45 & 67 & 1800 & 158 \\
\hline 4.0 & 56 & 84 & 1950 & 169 \\
\hline 1.0 & 63 & 94 & 2000 & 177 \\
\hline 1.0 & 65 & 97 & 1950 & 183 \\
\hline 1.0 & 70 & 105 & 2000 & 187 \\
\hline 2.0 & 74 & 111 & 1900 & 197 \\
\hline 1.0 & 84 & 126 & 2050 & 202 \\
\hline 1.0 & 85 & 128 & 1950 & 209 \\
\hline$\infty$ & 99 & 148 & 1950 & 225 \\
\hline
\end{tabular}

$$
T_{d}=k y+T_{0} .
$$

The obtained parameter $T_{0}$ equals $3.114 \mathrm{~s}$. This intercept corresponds to the distance $y=0$, representing the duration of a particle directly under the midline of the track. In other words, it is the duration for a train passing through a cross section of the roadbed, and thus, we herein refer $T_{0}$ as the train passing time. As shown in Figure 1, the distance between the front and the last axle of the urban train $L=76 \mathrm{~m}$. As the train passing time $T_{0}=3.114 \mathrm{~s}$ in this case, it can be inferred that the speed $c$ of the train at $7: 27$ is $L / T_{0}=24.4 \mathrm{~m} / \mathrm{s}$. We calculate all the vibration data in Table 3 by the regression analysis and list the obtained train passing times and vehicle speeds in Table 4.

The designed operating speed of the train (DKZ5) in the test is $22.22 \mathrm{~m} / \mathrm{s}$, but some calculated values in Table 4 are above the operating speed. As mentioned earlier, the test site was located between two stations. The train's arrival time at the stations was strictly specified, but during the journey between the two stations, the train underwent acceleration and deceleration. At the moment when the observation array was passed, the speeds of the trains are not of the same value. The average of the speeds in Table 4 obtained by the regression analysis is equal to $21.98 \mathrm{~m} / \mathrm{s}$, which is very close to the designed operating speed of the train.

There is no doubt that the instantaneous speed measured in the field is the ideal data to test the accuracy of this regression analysis method. Unfortunately, due to equipment reasons, field tests failed to obtain sufficiently meaningful data of the instantaneous vehicle speed. Many researchers (e.g., $[4,6,10])$ pointed out that higher vehicle speed results in more significant ground vibration intensity. Figure 8 shows the speculated vehicle speeds and the corresponding observed PGAs of P1, where the values have been normalized with those of the train $7: 27$. It can be seen that the speed by the regression analysis has a positive correlation with the observed vibration intensity, which is consistent with the general knowledge of the literature. This result may be deemed as a preliminary validation for the proposed regression method.

The tendency of the increase of duration with distance is related to the Doppler effect caused by the moving excitation source. Equation (11) indicates that the vibration time history consists of the vibration directly from the train's passing $\left(T_{0}\right)$ and the tailing portion caused by the Doppler effect. Figure 6 shows that the farther away from the track, the more pronounced the effect of this tailing diffusion. The coefficient $k$ in the regression equation (11) reflects the degree to which the duration increases with increasing distance. We plot the difference between the measured vibration duration and the train passing time in Table 3 into the coordinate system $\left(T_{d}-T_{0}, y\right)$, as shown in Figure 9. We can see that the observation data are concentrated in a narrow band, indicating that the variation of the slope $k$ is not significant and the average value is $0.082467 \mathrm{~s} / \mathrm{m}$. Therefore, as far as this observation site is concerned, the time history of the surface vibration caused by the Beijing Urban Rail Line 13 can be expressed as follows: 

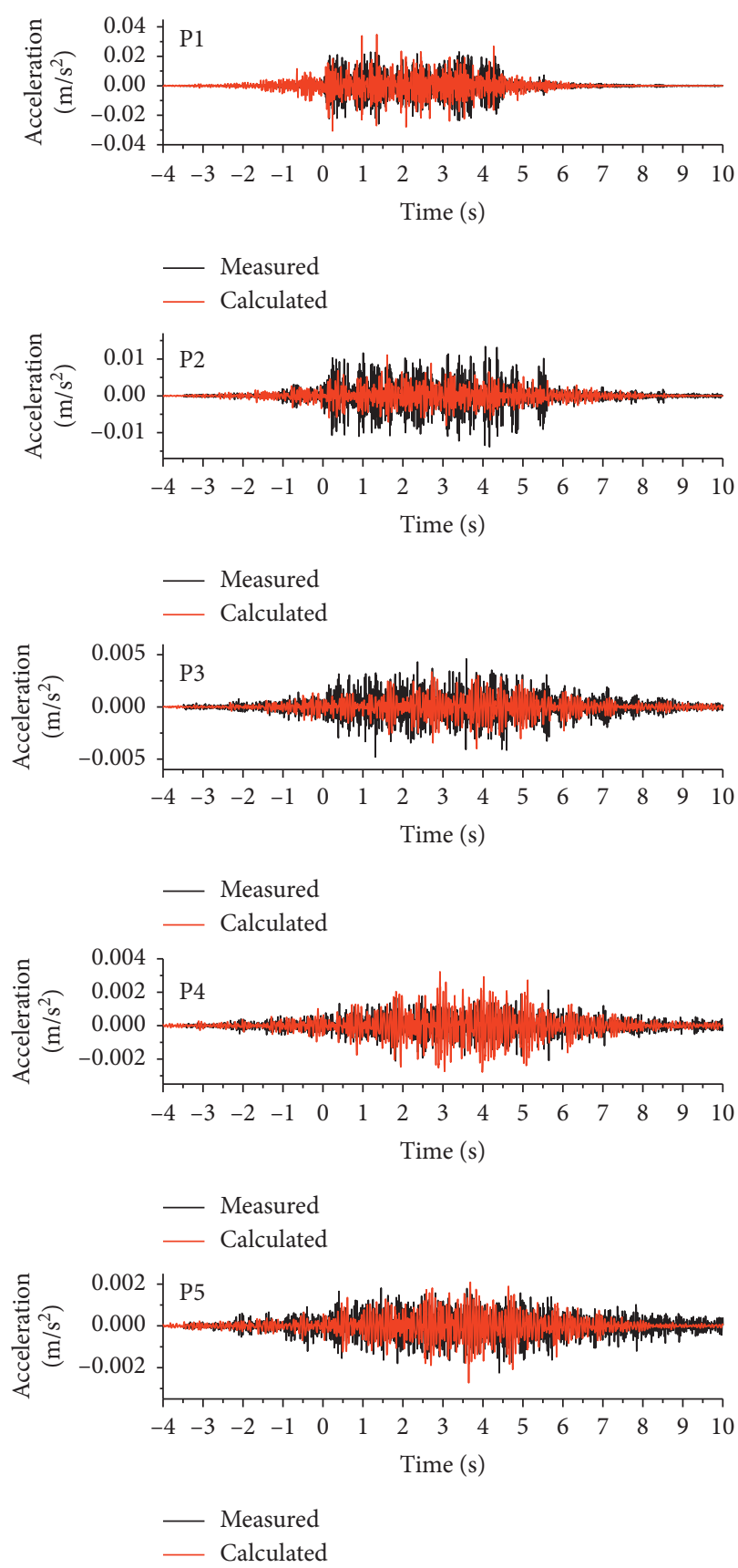

FIGURE 4: Comparison of calculated time histories to the measured data, recorded at 7:40 am on March 6, 2007.

$$
T_{d}=\frac{L}{c}+0.082467 y
$$

\section{Attenuation Feature of Vibration Intensity}

5.1. Ground Vibration Intensity. Table 3 shows that, compared with the two horizontal components, the vertical vibration PGA is the largest one, which indicates that the vertical component dominates the ground vibration. Therefore, we suggest to use the vertical component as the predominant index in the study of ground vibration caused by urban rail transit.
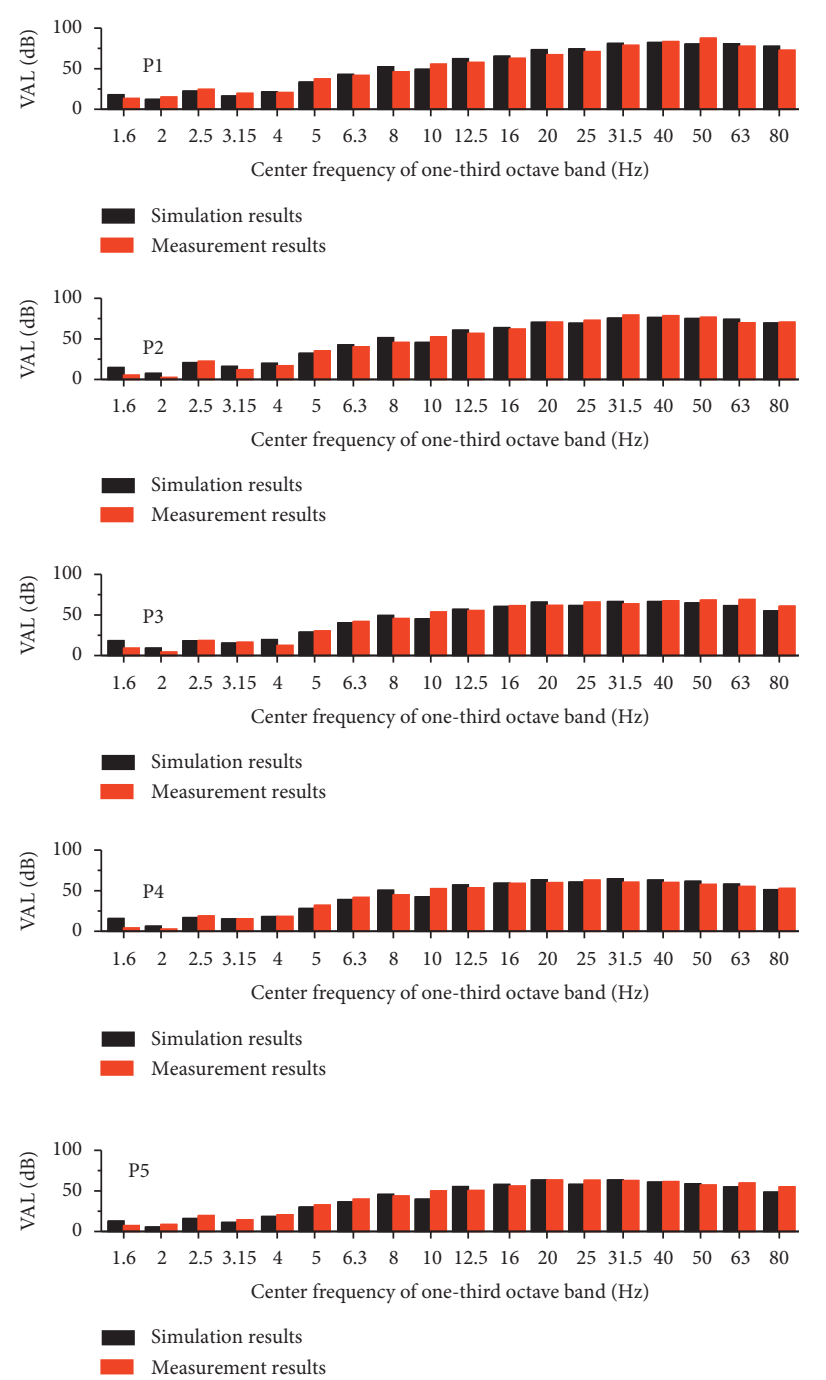

FIGURE 5: Comparison of calculated vibration acceleration levels to the measured results.

It can be found in Figure 6 that as the distance from the track increases, the vibration intensity gradually becomes smaller. The equation describing this gradual decrease is often referred to as the attenuation relationship. In rail transit planning, the attenuation relationship is one of the essential basis for environmental vibration prediction and vibration reduction design.

A reliable vibration attenuation relationship is based on a large number of observational tests and obtained through statistical analysis. At present, there is no generally accepted statistical attenuation relationship for ground-running urban rail transit. By taking account of the geometric damping and the material damping effect, Cui et al. [14] performed a regression analysis on the attenuation of vertical PGA. They put forward an attenuation formula as follows:

$$
A=0.0564 y^{-0.3126} e^{-0.0285 y},
$$

where $A$ is the PGA $\left(\mathrm{m} / \mathrm{s}^{2}\right)$ of the ground vibration and $y$ is the distance $(\mathrm{m})$ from the observation point to the midline of the track. 


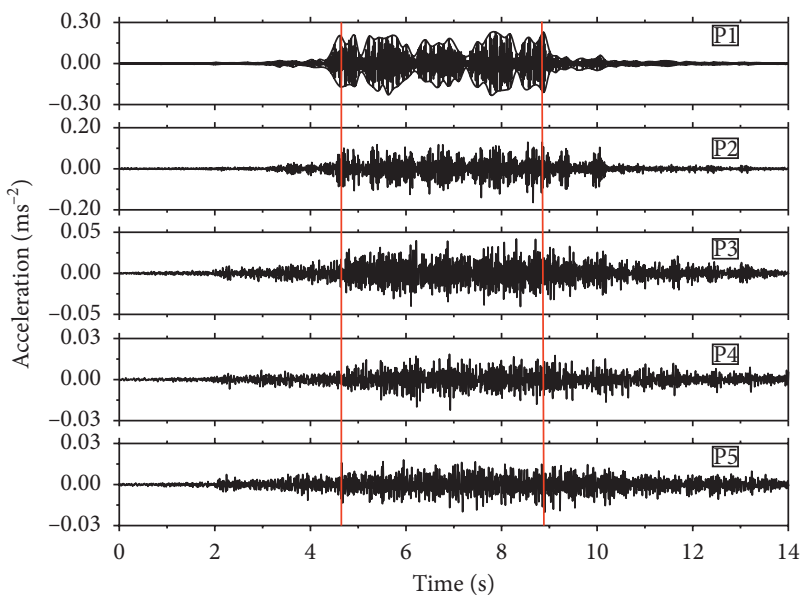

Figure 6: Measured acceleration time histories at the 5 points, recorded at 7:27 am on March 6, 2007.

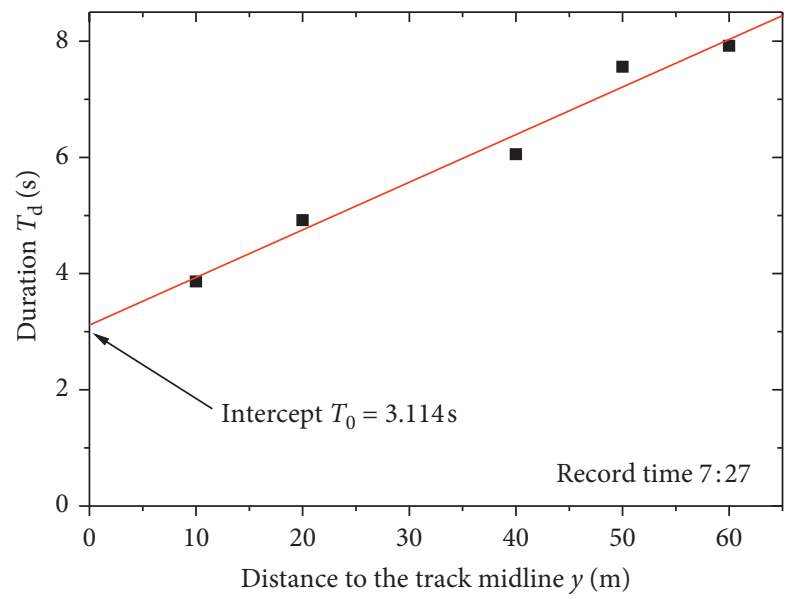

FIgURE 7: Variation of duration with distance to the track.

TABLE 3: Durations and PGAs of vibrations recorded at all 5 points.

\begin{tabular}{|c|c|c|c|c|c|}
\hline \multirow{2}{*}{ Record time } & \multirow{2}{*}{ Distance to track (m) } & \multicolumn{3}{|c|}{ PGA $\left(\mathrm{m} / \mathrm{s}^{2}\right)$} & \multirow{2}{*}{ Duration (s) } \\
\hline & & $x$-component & $y$-component & $z$-component & \\
\hline \multirow{5}{*}{$7: 27$} & 10 & $1.55 E-02$ & $9.03 E-03$ & $3.08 E-02$ & 3.865 \\
\hline & 20 & $1.08 E-02$ & $7.13 E-03$ & $1.72 E-02$ & 4.920 \\
\hline & 40 & $2.34 E-03$ & $2.68 E-03$ & $5.33 E-03$ & 6.055 \\
\hline & 50 & $2.09 E-03$ & $1.64 E-03$ & $2.52 E-03$ & 7.560 \\
\hline & 60 & $2.12 E-03$ & $2.54 E-03$ & $3.89 E-03$ & 7.920 \\
\hline \multirow{5}{*}{$7: 34$} & 10 & $1.20 E-02$ & $8.92 E-03$ & $2.34 E-02$ & 4.160 \\
\hline & 20 & $8.64 E-03$ & $7.84 E-03$ & $1.68 E-02$ & 5.285 \\
\hline & 40 & $2.56 E-03$ & $2.55 E-03$ & $4.27 E-03$ & 7.055 \\
\hline & 50 & $1.75 E-03$ & $1.62 E-03$ & $2.28 E-03$ & 7.975 \\
\hline & 60 & $1.27 E-03$ & $1.80 E-03$ & $2.06 E-03$ & 8.730 \\
\hline \multirow{5}{*}{$7: 40$} & 10 & $1.21 E-02$ & $1.00 E-02$ & $2.59 E-02$ & 4.105 \\
\hline & 20 & $9.41 E-03$ & $8.46 E-03$ & $1.38 E-02$ & 5.200 \\
\hline & 40 & $2.67 E-03$ & $2.41 E-03$ & $4.79 E-03$ & 6.645 \\
\hline & 50 & $1.59 E-03$ & $1.36 E-03$ & $2.12 E-03$ & 7.620 \\
\hline & 60 & $1.30 E-03$ & $2.07 E-03$ & $2.25 E-03$ & 8.450 \\
\hline
\end{tabular}


TABLE 3: Continued.

\begin{tabular}{|c|c|c|c|c|c|}
\hline \multirow{2}{*}{ Record time } & \multirow{2}{*}{ Distance to track (m) } & \multicolumn{3}{|c|}{ PGA $\left(\mathrm{m} / \mathrm{s}^{2}\right)$} & \multirow{2}{*}{ Duration $(\mathrm{s}$} \\
\hline & & $x$-component & $y$-component & $z$-component & \\
\hline \multirow{5}{*}{$7: 46$} & 10 & $1.24 E-02$ & $6.28 E-03$ & $2.16 E-02$ & 4.700 \\
\hline & 20 & $8.06 E-03$ & $5.23 E-03$ & $1.19 E-02$ & 5.945 \\
\hline & 40 & $2.41 E-03$ & $2.57 E-03$ & $4.39 E-03$ & 7.745 \\
\hline & 50 & $1.70 E-03$ & $1.33 E-03$ & $2.01 E-03$ & 8.930 \\
\hline & 60 & $1.40 E-03$ & $1.78 E-03$ & $2.80 E-03$ & 9.350 \\
\hline \multirow{5}{*}{$7: 53$} & 10 & $1.41 E-02$ & $9.95 E-03$ & $2.71 E-02$ & 3.885 \\
\hline & 20 & $8.44 E-03$ & $6.03 E-03$ & $1.42 E-02$ & 4.955 \\
\hline & 40 & $2.43 E-03$ & $2.15 E-03$ & $4.84 E-03$ & 6.895 \\
\hline & 50 & $1.77 E-03$ & $1.65 E-03$ & $2.41 E-03$ & 8.270 \\
\hline & 60 & $1.62 E-03$ & $2.14 E-03$ & $2.13 E-03$ & 8.770 \\
\hline \multirow{5}{*}{$10: 32$} & 10 & $1.34 E-02$ & $8.52 E-03$ & $2.32 E-02$ & 4.130 \\
\hline & 20 & $1.16 E-02$ & $6.92 E-03$ & $1.65 E-02$ & 5.665 \\
\hline & 40 & - & - & - & - \\
\hline & 50 & $2.51 E-03$ & $1.60 E-03$ & $3.04 E-03$ & 8.420 \\
\hline & 60 & $3.46 E-03$ & $3.20 E-03$ & $3.13 E-03$ & \\
\hline \multirow{5}{*}{$10: 38$} & 10 & $1.30 E-02$ & $6.92 E-03$ & $2.67 E-02$ & 3.945 \\
\hline & 20 & $1.06 E-02$ & $5.20 E-03$ & $1.91 E-02$ & 5.010 \\
\hline & 40 & - & - & - & - \\
\hline & 50 & $1.61 E-03$ & $1.45 E-03$ & $2.20 E-03$ & 7.260 \\
\hline & 60 & - & - & - & - \\
\hline \multirow{5}{*}{$11: 00$} & 10 & $1.13 E-02$ & $6.12 E-03$ & $2.22 E-02$ & 4.335 \\
\hline & 20 & $1.30 E-02$ & $4.87 E-03$ & $1.72 E-02$ & 5.520 \\
\hline & 40 & $2.37 E-03$ & $2.96 E-03$ & $4.81 E-03$ & 7.075 \\
\hline & 50 & $2.17 E-03$ & $1.05 E-03$ & $2.03 E-03$ & 8.095 \\
\hline & 60 & $1.61 E-03$ & $1.80 E-03$ & $2.11 E-03$ & 8.220 \\
\hline \multirow{5}{*}{$11: 15$} & 10 & - & - & - & - \\
\hline & 20 & $1.31 E-02$ & $6.53 E-03$ & $1.82 E-02$ & 5.045 \\
\hline & 40 & $2.78 E-03$ & $2.20 E-03$ & $4.84 E-03$ & 6.575 \\
\hline & 50 & $1.58 E-03$ & $1.20 E-03$ & $2.60 E-03$ & 7.240 \\
\hline & 60 & $1.77 E-03$ & $1.85 E-03$ & $2.98 E-03$ & 7.230 \\
\hline \multirow{5}{*}{$11: 25$} & 10 & - & - & - & - \\
\hline & 20 & $1.32 E-02$ & $5.79 E-03$ & $2.07 E-02$ & 5.050 \\
\hline & 40 & $2.63 E-03$ & $2.55 E-03$ & $5.28 E-03$ & 6.435 \\
\hline & 50 & $1.77 E-03$ & $1.37 E-03$ & $2.58 E-03$ & 7.360 \\
\hline & 60 & $1.84 E-03$ & $2.05 E-03$ & $2.78 E-03$ & 7.340 \\
\hline
\end{tabular}

TABle 4: Train passing time and train speed obtained by regression.

\begin{tabular}{lcccc}
\hline Record time & Train passing time $T_{0}(\mathrm{~s})$ & Slope $k$ & $R^{2}$ & Train speed $(\mathrm{m} / \mathrm{s})$ \\
\hline $7: 27$ & 3.114 & 0.0819 & 0.9684 & 24.40 \\
$7: 34$ & 3.362 & 0.0911 & 0.9964 & 22.61 \\
$7: 40$ & 3.345 & 0.0850 & 0.9954 & 22.72 \\
$7: 46$ & 3.921 & 0.0948 & 0.9842 & 19.38 \\
$7: 53$ & 2.923 & 0.1009 & 0.9894 & 26.00 \\
$10: 32$ & 3.307 & 0.1037 & 0.9721 & 22.98 \\
$10: 38$ & 3.243 & 0.0811 & 0.9880 & 23.43 \\
$11: 00$ & 3.763 & 0.0802 & 0.9629 & 18.76 \\
$11: 15$ & 4.051 & 0.0581 & 0.8861 & 19.36 \\
$11: 25$ & 3.926 & 0.0616 & 0.9075 & \\
\hline
\end{tabular}

Figure 10(a) shows the decaying curve of equation (13) and a set of measured data of PGA as well as the calculation results from the present train-track-ground model. At the five observation points, equation (13) is in good agreement with the measured data. Especially at the $10 \mathrm{~m}$ point, where the mathematical model appears relative conservative, the result of the attenuation equation (13) is even closer to the data than the value calculated by the model.

The numerical results of the model are more abundant than those from the experiment, showing the overall trend of 


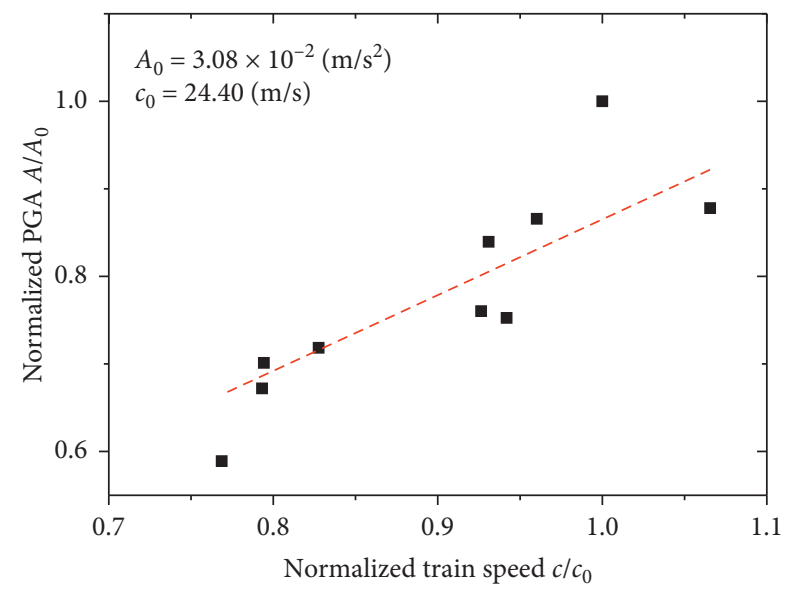

FIGURE 8: Correlation between peak ground acceleration and train speed.

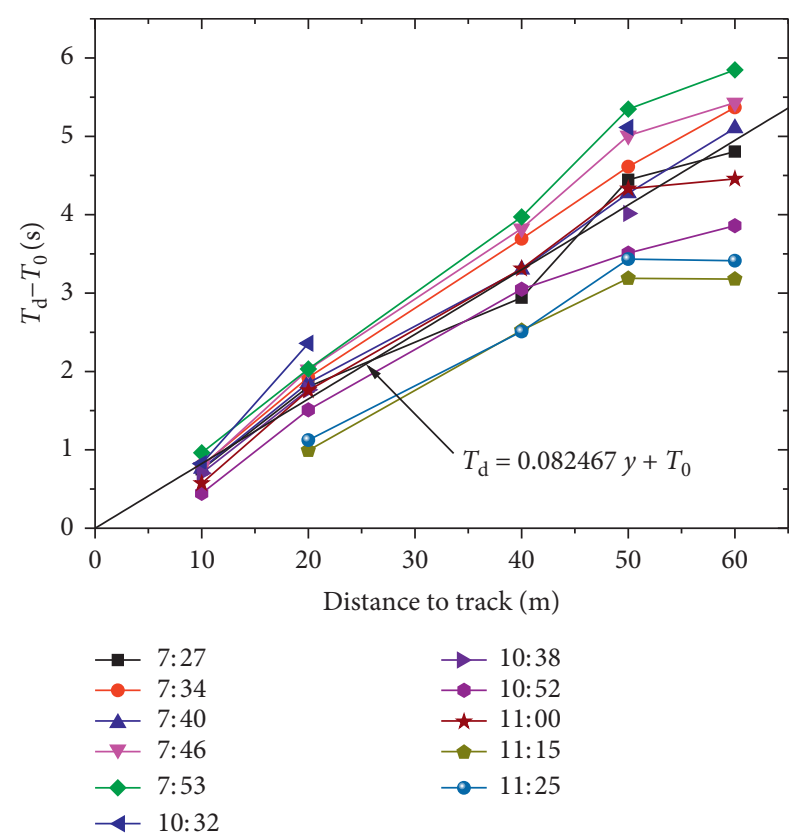

Figure 9: Fitting of duration increase with distance.

attenuation and some local details. As the distance increases, the vibration decays as a whole, whereas two local amplifications occur at $20 \mathrm{~m}$ and $38 \mathrm{~m}$ away, respectively.

It should be mentioned that because the local amplification phenomenon is related to the superposition of reflected waves and refracted waves at soil layer interfaces $[26,27]$, the amplification and its location are significantly different for grounds with different soil structures. An attenuation relationship is an average result from a large number of grounds and usually cannot express the local amplification effect of a specific site. Therefore, it is inevitable that the curve of equation (13) deviates from the simulation result in the range of $20-40 \mathrm{~m}$ in the figure.

Within the range of $0-10 \mathrm{~m}$, however, the value of equation (13) is far lower than that of the numerical simulation, which suggests that this attenuation relationship may give an over-optimistic prediction of the vibration.
Besides, when reaching the midline of the track, $y$ is approaching zero, which makes the result of equation (13) go to infinity. This is also not realistic. The figure shows that, after entering the subgrade just below the embankment, the numerical simulation reaches a maximum and no longer grows. Based on the simulation, we propose herein an additional correction to equation (13) as follows:

$$
A= \begin{cases}A_{0}, & y<b, \\ \frac{(y-10)\left(A_{0}-0.0206\right)}{b-10}+0.0206, & b \leq y<10 \mathrm{~m} \\ 0.0564 y^{-0.3126} e^{-0.0285 y}, & y \geq 10 \mathrm{~m}\end{cases}
$$

where the defined distance $b$ is the half-width of the embankment bottom and $A_{0}$ is the average PGA of the subgrade. Figure 10(b) shows the curve of the piecewise function (14). One can see that within the range of $0-10 \mathrm{~m}$, the value of the modified attenuation relationship is higher than that of the original equation (13). At the midline of the subgrade, equation (14) has a maximum limit to eliminate the singularity of equation (13).

5.2. Vibration Intensity of Subgrade. For the sake of operational safety, it is usually not allowed to deploy sensors in the subgrade of the track in service. Numerical simulation is an acceptable supplement to explore the subgrade vibration varying with the depth. Figure 11 shows the numerical results of vertical stress. The abscissa denotes the maximum value of the vertical stress time history, while the ordinate is the depth from the bottom of the embankment, with the downward direction being positive. To emphasize generality, we normalize the stress with the value at the origin $x=y=z=0$ (i.e., the midline of the embankment bottom). We can see that, as the depth increases, the dynamic stress level decays exponentially:

$$
\frac{\sigma}{\sigma_{0}}=c_{1}+c_{2} e^{-c_{3} z}
$$

where $c_{1}, c_{2}$, and $c_{3}$ are three fitting parameters. For the site of this in situ test, $c_{1}=0.11756, c_{2}=0.87414$, and $c_{3}=0.58640$.

Figure 12 is the distribution of the vertical acceleration peak of the subgrade with the depth, where the abscissa represents the normalized value $A / A_{0}$ and $A_{0}$ is the peak acceleration of the particle at the origin. The graph shows a monotonic attenuation that is very fast and exhibits exponential behavior as follows:

$$
\frac{A}{A_{0}}=e^{-c z} \text {. }
$$

For this site, the fitting parameter $c=1.1662$.

\section{Characteristic of Wheel/Rail Interactive Force}

At the moving wheel/rail contact, there is a wheel/rail interactive force that dynamically changes in magnitude over time. Therefore, the wheel/rail force is a moving and 


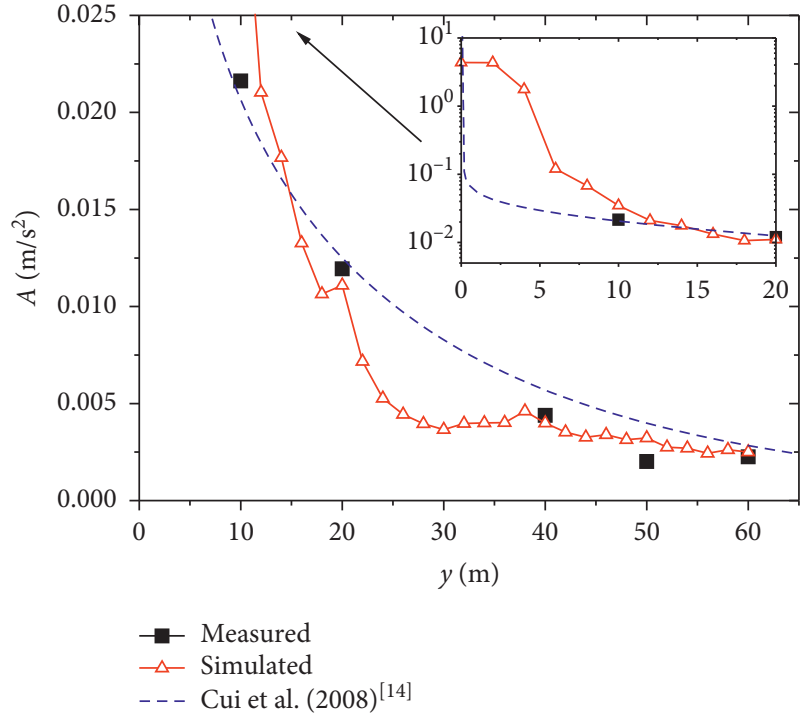

(a)

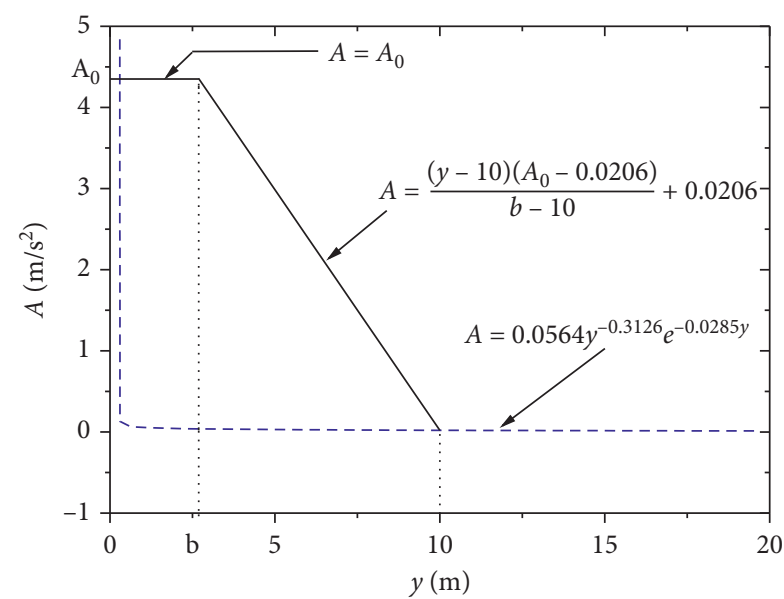

(b)

FIGURE 10: Attenuation of ground vibration intensity: (a) attenuation feature; (b) attenuation relationship.

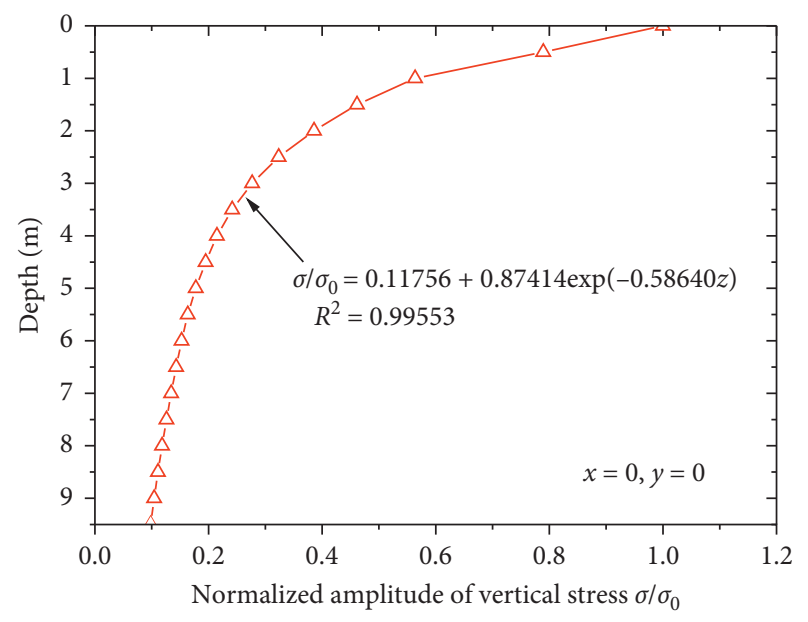

Figure 11: Variation of soil stress amplitude with depth in subgrade.

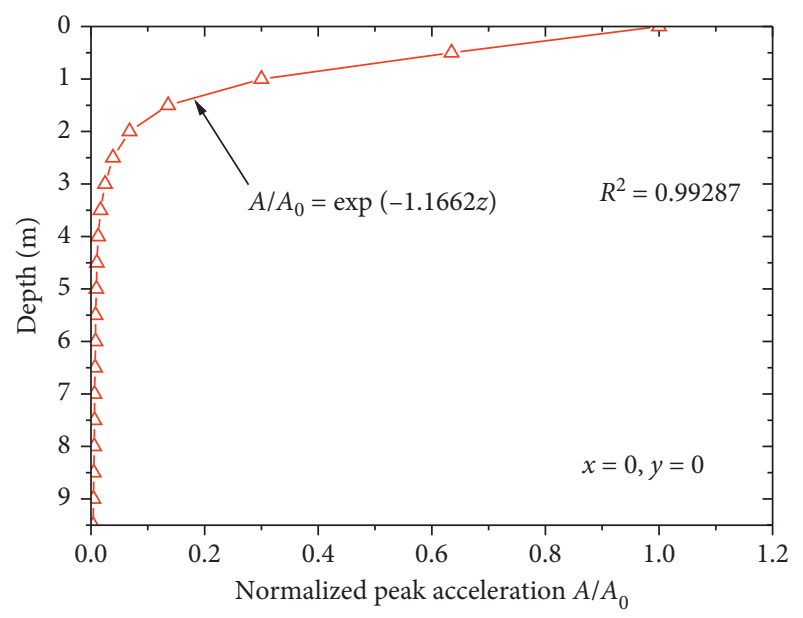

Figure 12: Peak acceleration of subgrade decaying with depth. dynamic load. Figure 13(a) shows a time history of the wheel/rail force calculated by the present train-track-ground model. One can see that unlike the time histories of the soil particles in Figure 4, the wheel/rail force shows stationary feature and a certain periodicity. Wheel/rail forces move with a train and are quantities observed from the perspective of an observer on the train. As long as the train moves, the dynamic wheel/rail force always exists. On the contrary, the mass point of track or soil exists on the earth. Therefore, its vibration first increases when a train coming and then decreases when the train is leaving, showing a typical nonstationary feature. The results of Figure 13(a) reflect the stationarity nature of the wheel/rail interaction, with the values fluctuating around the quasi-static axle weight of $146 \mathrm{kN}$.

Figure 13(b) shows the power spectrum of the wheel/ rail interaction. Overall, as the frequency increases, the dynamic wheel/rail force decreases. The predominant frequency range is $12-14 \mathrm{~Hz}$, indicating that the content in this band plays a significant role in the time history of Figure 13(a).

The dynamic wheel/rail force is related to three factors. The first one is the train. As the vertical excitation frequency increases, the vertical vibration amplitude of the wheel decreases and therefore the contribution to the wheel/rail force decreases. The second factor is the wheel/rail uneven contact. When a train moves along a track, the unevenness of various wavelengths stimulates wheel/rail forces at the corresponding frequencies. The shorter the wavelength, the higher the frequency. For rail transit, the shorter the uneven wavelength, the smaller the amplitude, and thus the smaller the exciting wheel/rail force. Therefore, the overall decreasing tendency of Figure 13(b) reflects both the effects of wheel/rail unevenness and trains. The third factor is the track-ground system. Figure 14 is the dynamic receptance of the track-ground, with a single peak showing around $14 \mathrm{~Hz}$. 


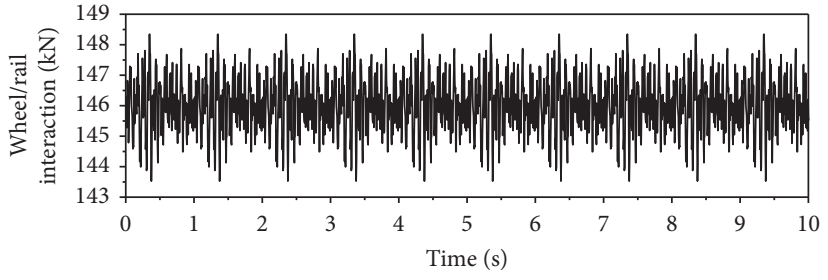

(a)

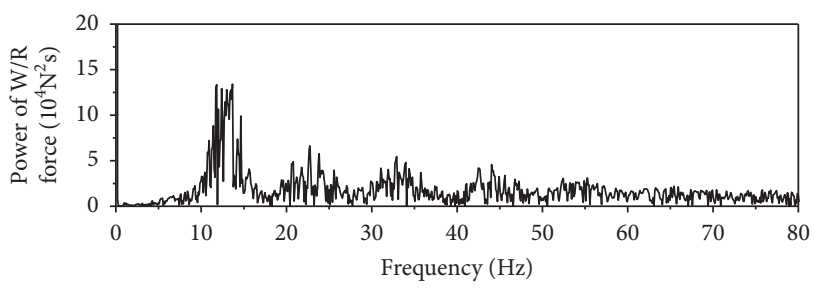

(b)

FIGURE 13: Simulated wheel/rail interaction force: (a) time history; (b) power spectrum.

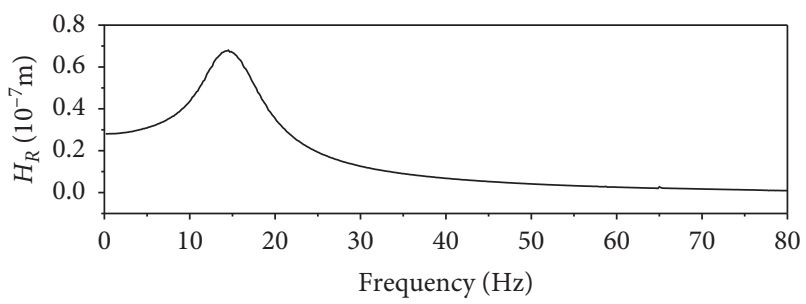

FIGURE 14: Receptance of track-ground system.

By comparing it with Figure 13(b), we find that the predominant frequency band in Figure 13(b) is the effect of the track-ground system.

\section{Conclusion}

The urban rail transit system includes a train in a moving reference system and a track-subgrade-surrounding soil in the fixed reference system of the earth. The vibration problem involves the soil-structure dynamic interaction in the two reference frames. The system is so complicated that the induced vibration needs to be further studied. In this research, by employing the field measurement and numerical simulation, we reveal the duration and intensity characteristics of the ground and subgrade vibrations. A regression analysis method is proposed for identifying train passing time and running speed from data of ground vibration. An attenuation relationship of peak ground acceleration is verified and improved, and the attenuation in the depth direction is revealed as well.

The analysis of the measured data shows that the ground vibration duration is composed of two parts. One part is the train passing time that is irrelevant with the position of the observation point, while the other is the tailing duration that increases with the distance from the track. As far as a specific site is concerned, the rate of increase of tailing duration with distance varies little. A linear function can describe the relationship between duration and distance, and the intercept is equal to the train passing time. The method may provide a reference for parameter identification problems of moving sources such as ships, automobiles, and airplanes.

Among the three directions of the ground vibration, the vertical vibration intensity is the largest. For the study of the surface environmental vibration caused by urban rail transit, we recommend using the vertical component as the predominant index. The intensity of ground vibration decays with the distance from the track. We propose a piecewise function to express the attenuation relationship.

The vertical dynamic stress intensity of the subgrade gradually decreases downward, and the attenuation is exponential. The peak acceleration also decreases exponentially, and the attenuation is more rapid than that of stress.

The wheel/rail interaction behaves as a stationary random process that has a certain periodicity. The value dynamically varies around the gravity distributed by the axles. Further physical model tests and numerical simulations may use the time history of the wheel/rail force as a sample of random excitation.

\section{Data Availability}

The data used to support the findings of this study are available from the corresponding author upon request.

\section{Conflicts of Interest}

The authors declare that they have no conflicts of interest.

\section{Acknowledgments}

This research was supported by the National Natural Science Foundation of China (Grant nos. 50538030 and 51678540), Postdoctoral Science Foundation of China (Grant no. 2013M531084), and Natural Science Foundation of Heilongjiang Province of China (Grant no. E201221).

\section{References}

[1] H. Xia, N. Zhang, and Y. M. Cao, "Experimental study of train-induced vibrations of environments and buildings," Journal of Sound and Vibration, vol. 280, no. 3-5, pp. 10171029, 2005.

[2] J. Chen, H. Xia, S. Chen, and M. Su, "Investigation on running-train-induced ground vibrations near railway," Engineering Mechanics, vol. 27, no. 1, pp. 98-103, 2010, in Chinese.

[3] G. Gao, Z. Li, S. Feng, and Y. Sun, "Experimental results and numerical predictions of ground vibration induced by highspeed train running on Qin-Shen railway," Rock and Soil Mechanics, vol. 28, no. 9, pp. 1817-1822, 2007, in Chinese.

[4] K. Mao, G. Chen, and H. Zhuang, "Parametric analysis of ground surface vibration induced by elevated rail transit," Rock and Soil Mechanics, vol. 33, no. 11, pp. 3400-3406, 2012, in Chinese.

[5] S. Chang, H. Bai, J. Ye, and Z. Zhang, "Dynamic response of railway sandy foundation uder train running loading," 
Engineering Geology, vol. 22, no. 6, pp. 1077-1085, 2014, in Chinese.

[6] L. Auersch, "Ground vibration due to railway traffic-The calculation of the effects of moving static loads and their experimental verification," Journal of Sound and Vibration, vol. 293, no. 3-5, pp. 599-610, 2006.

[7] S.-J. Feng, X.-L. Zhang, Q.-T. Zheng, and L. Wang, "Simulation and mitigation analysis of ground vibrations induced by high-speed train with three dimensional FEM," Soil Dynamics and Earthquake Engineering, vol. 94, pp. 204-214, 2017.

[8] L. Tian, X. Ling, L. Wang, F. Zhang, and S. Chen, "Study on vibration response of railway subgrade in seasonally frozen region induced by train-streering in winder," World Eathquake Engineering, vol. 30, no. 4, pp. 34-40, 2014, in Chinese.

[9] J. Dong and Z. Wu, "Propagation laws of heavy-haul trainsinduced ground vibration in seasonal frozon area of north China plain," Journal of Vibration and Shock, vol. 37, no. 23, pp. 286-291, 2018, in Chinese.

[10] Y. Tang, Q. Yang, X. Ren, and S. Xiao, "Dynamic response of soft soils in high-speed rail foundation: in situ measurements and time domain finite element method model," Canadian Geotechnical Journal, vol. 56, no. 12, pp. 1832-1848, 2019.

[11] J. Hu, Y. Luo, and J. Xu, "Experimental and numerical analysis and prediction of ground vibrations due to heavy haul railway viaduct," Mathematical Problems in Engineering, vol. 2019, Article ID 2751815, 15 pages, 2019.

[12] W. Liu, W. Liu, S. Gupta, and G. Degrand, "Prediction of vibrations in the tunnel and free field due to passage of metro trains," Engineering Mechanics, vol. 27, no. 1, pp. 250-256, 2010, in Chinese.

[13] P. Lu, T. Liu, Z. Zhang, X. Li, and S. Sun, "Simulation Study on the effect of damping thickness of viscoelastic damping track bed on tunnel and surface vibratoin attenuation characteristcs," Railway Standard Design, vol. 63, no. 3, pp. 49-54, 2019, in Chinese.

[14] G. Cui, X. Tao, and X. Chen, "Least-square criterion based studies on environmental vibration induced by urban rail transit on ground," Journal of Harbin Institute of Technology, vol. 40, no. 8, pp. 1184-1188, 2008, in Chinese.

[15] F. Wang, X. Tao, G. Cui, X. Zheng, and D. Liang, "Test in situ for free ground vibration near urban railway line," Journal of Vibration and Shock, vol. 30, no. 5, pp. 131-135, 2011, in Chinese.

[16] F. Wang, X. Tao, and X. Zheng, "Inversion of excitation source in ground vibration from urban railway traffic," Science China Technological Sciences, vol. 55, no. 4, pp. 950-959, 2012.

[17] X. Zheng, X. X. Tao, F. T. Wang et al., "An auto-cross PSD method to remove background vibration from observational records of traffic environmental vibration," Chinese Journal of Geophysics, vol. 56, no. 1, pp. 348-353, 2013, in Chinese.

[18] F. T. Wang, X. X. Tao, X. Zheng et al., "PSD correction method for removing background vibration from traffic environmental vibration observation," Journal of Vibration and Shock, vol. 30, no. 4, pp. 124-172, 2011, in Chinese.

[19] X. D. Zhang, W. M. Yan, M. Ren, and M. Liu, "Background vibration analysis for traffic environmental vibration testing," Journal of Vibration and Shock, vol. 28, no. 4, pp. 177-212, 2009, in Chinese.

[20] X. Sheng, C. J. C. Jones, and D. J. Thompson, "A theoretical model for ground vibration from trains generated by vertical track irregularities," Journal of Sound and Vibration, vol. 272, no. 3-5, pp. 937-965, 2004.

[21] F. Wang, Inversioin of Ground Vibration Source by Urban Railway Traffic in Frequency Domain, Harbin Institute of Technology, Harbin, China, 2011, in Chinese.
[22] F. Wang, X. Tao, L. Xie et al., "Dynamic Green's function of stratified ground with saturated soil layers for modeling ground vibration near railway track," Chinese Journal of Geophysics-Chinese Edition, vol. 58, no. 8, pp. 2948-2961, 2015, in Chinese.

[23] F. Wang, X. Tao, L. Xie, and S. Raj, “Green's function of multilayered poroelastic half-space for models of ground vibration due to railway traffic," Earthquake Engineering and Engineering Vibration, vol. 16, no. 2, pp. 311-328, 2017.

[24] X. Zheng, Demonstration of Source Function Inverstion of Vibration Caused by Urban Rail Traffic, Harbin Institute of Technology, Harbin, China, 2012, in Chinese.

[25] X. X. Tao, X. Zheng, and F. T. Wang, "Excitation source function of ground vibration from urban railway traffic by an inversion procedure," Applied Mechanics and Materials, vol. 226-228, pp. 374-380, 2012.

[26] H. M. Liu, X. X. Tao, X. Zheng, and F. T. Wang, “An explanation on mechanism of local amplification in attenuation of ground vibration from rail traffic in urban area," Applied Mechanics and Materials, vol. 226-228, pp. 392-395, 2012.

[27] X. Zheng, X. Tao, F. Wang, and L. Xie, "Mechanism of local amplication in attenuation of ground vibration induced by rail traffic," Journal of Vibration and Shock, vol. 33, no. 3, pp. 35-40, 2014, in Chinese.

[28] X. Zheng, Y. Zhang, X. X. Tao, F. T. Wang, and L. L. Xie, "Research on model calculating depth of environmental vibration caused by the ground rail traffic load," Advanced Materials Research, vol. 912-914, pp. 1698-1701, 2014.

[29] The State Standard of the People's Repulic of China, Measurement Method of Environmental Vibration of Urban Area (GB10070-88), The State Standard of the People's Repulic of China, China, 1988, in Chinese.

[30] Y. Hu, Earthquake Engineering, Seismological Press, Beijing, China, 2006, in Chinese, 2nd edition. 